Meta

Journal des traducteurs

Translators' Journal

\title{
Dépouillement de corpus à des fins terminologiques dans un univers dépendant du temps
}

\section{Uzoma Chukwu}

Volume 43, numéro 3, septembre 1998

URI : https://id.erudit.org/iderudit/002243ar

DOI : https://doi.org/10.7202/002243ar

Aller au sommaire du numéro

\section{Éditeur(s)}

Les Presses de l'Université de Montréal

ISSN

0026-0452 (imprimé)

1492-1421 (numérique)

Découvrir la revue

Citer cet article

Chukwu, U. (1998). Dépouillement de corpus à des fins terminologiques dans un univers dépendant du temps. Meta, 43(3), 411-425.

https://doi.org/10.7202/002243ar
Résumé de l'article

Cet article traite du problème de dépouillement de corpus à des fins de constitution de la nomenclature du dictionnaire terminologique par le terminologue-linguiste fonctionnant dans un univers dont le temps est une composante. Il pose que le seul problème qui puisse réellement empêcher l'aboutissement de l'exercice est d'ordre motivationnel et, ainsi, ramène le problème à celui de la maîtrise du temps motivationnel. Il propose, enfin, la recherche de cette maîtrise dans le cadre du pacte terminologique, une "grammaire de haut niveau" du texte de spécialité, capable non seulement de prédire les unités susceptibles d'être des termes et des configurations textuelles susceptibles d'en contenir, mais aussi de fournir des tests de terminologisme. 


\title{
ÉTUDES TERMINOLOGIQUES ET LINGUISTIQUES
}

\section{DÉPOUILLEMENT DE CORPUS À DES FINS TERMINOLOGIQUES DANS UN UNIVERS DÉPENDANT DU TEMPS}

\begin{abstract}
Résumé
Cet article traite du problème de dépouillement de corpus à des fins de constitution de la nomenclature du dictionnaire terminologique par le terminologue-linguiste fonctionnant dans un univers dont le temps est une composante. Il pose que le seul problème qui puisse réellement empêcher l'aboutissement de l'exercice est d'ordre motivationnel et, ainsi, ramène le problème à celui de la maitrise du temps motivationnel. Il propose, enfin, la recherche de cette maitrise dans le cadre du pacte terminologique, une «grammaire de haut niveau» du texte de spécialité, capable non seulement de prédire les unités susceptibles d'être des termes et des configurations textuelles susceptibles d'en contenir, mais aussi de fournir des tests de terminologisme.
\end{abstract}

\begin{abstract}
This paper discusses the problem of corpus analysis in the constitution of the nomenclature of terminological dictionaries by the linguist-terminologist, working in a time-dependent universe. It posits that the numerous problems associated with this task can be readily summed up as motivational and, therefore, to the urge to beat motivational time. It finally proposes a possible solution through the terminology pact, a "high-level grammar of specialized discourse" capable, on the one hand, of predicting the lexical forms of a text that are probable terms and text configurations that are likely to contain them and, on the other, of providing tests for terminological judgments.
\end{abstract}

\section{INTRODUCTION}

La formulation même de notre titre annonce des prises de position qui ne laisseront pas indifférent. Nous aurons l'occasion de revenir sur ces positions pour les argumenter. En attendant, il convient de souligner qu'alors que dans les années 1970 encore le problème du repérage des termes (et le problème connexe du découpage des termes) était un leitmotiv des discussions terminologiques, aujourd'hui, il semble de moins en moins figurer parmi les préoccupations des terminologues. Même un ouvrage aussi important que celui de Sager (1990) le passe complètement sous silence. Lorsque la question est abordée dans la littérature, c'est souvent dans le cadre de l'automatisation de la tâche. Il est certes difficile d'apporter des réponses définitives aux problèmes soulevés par le repérage des termes sans faire intervenir l'informatique. Cependant, on reconnaîtra aussi que pendant de longues années encore, cette tâche sera exécutée manuellement par le terminologue humain et que toute tentative d'automatisation qui ne fait pas du terminologue humain son modèle a très peu de chances d'aboutir.

Ce silence est d'autant plus étonnant que la part sans cesse croissante de ce qu'on a appelé la «terminologie descriptive», par opposition à la «terminologie de désignation» (voir Béjoint 1993), aurait incité à s'attendre au contraire. Doit-on en conclure que la tâche ne pose plus de problème ? Tant s'en faut, à notre avis. Il faut plutôt croire que c'est un problème qui soulève des questions qui, pour la plupart, ne relèvent pas du champ d'investigation habituel du terminologue, comme nous essaierons de le montrer ici.

Meta, XLIII, 3, 1998 


\section{PROBLÉMATISATION DU REPÉRAGE DES TERMES}

Posons le problème en termes plus concrets en imaginant les démarches sousjacentes à la résolution du problème par le terminologue humain naviguant à vue, c'està-dire se contentant d'une démarche générer-et-évaluer séquentielle simple. L'algorithme sous-jacent à une telle démarche pourrait se présenter de la sorte :

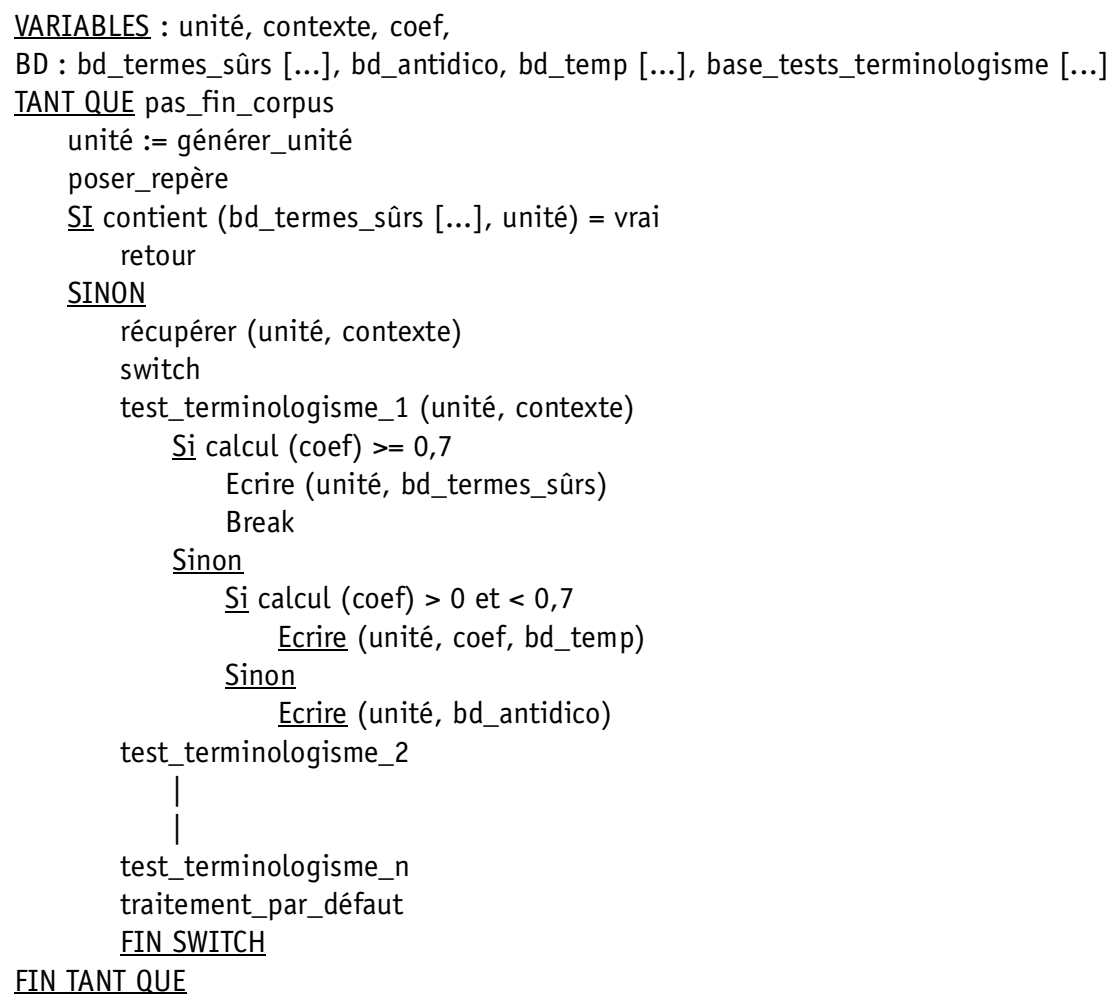

Notre algorithme n'est certainement pas le plus optimisé imaginable mais il nous semble offrir la garantie d'une solution au problème posé, à savoir le repérage exhaustif des termes du domaine cible si ceux-ci sont tous employés dans les textes du corpus. Cependant, la garantie ici ne vaut que pour un espace dont le temps n'est pas une composante. Très naturellement, quand on parle de temps, ici, on pense d'abord au «temps» observable physique. Ce raisonnement n'est pas faux, puisque la résolution de tout problème ne peut avoir lieu que dans le temps ${ }^{1}$. Mais nous n'allons pas nous en tenir là pour ne pas occulter les vrais problèmes. Un exemple ici éclaircirait mieux nos propos. Lorsqu'en matière de résolution machine de problèmes on distingue entre des problèmes qui peuvent être résolus en temps computationnel et ceux qui ne le peuvent, il nous semble que ce qui est en jeu est moins le temps (observable physique) que des ressources machine et, en l'occurrence, de la mémoire. Un problème qui ne peut pas être résolu en temps computationnel est donc un problème dont la résolution nécessite la création d'une pile qui ne peut pas tenir en mémoire. (Le lecteur se rappellera que la machine de Turing est dotée d'une mémoire infinie.) Partant de ces éclaircissements, la question est donc la suivante : le problème du repérage exhaustif des termes par le terminologue humain naviguant à vue peut-il être résolu en temps motivationnel ? 
Avant de tenter de répondre à cette question, essayons d'isoler les interrogations spécifiques permettant de prendre la mesure exacte du problème. Nous aimerions les ramener aux cinq suivantes :

1) Comment parcourir facilement un corpus de plusieurs centaines, voire de plusieurs milliers de pages?

2) Comment gérer un espace de recherche pour chaque terme potentiel du domaine quand on sait que cet espace est toujours égal à la taille du corpus ?

3) Comment gérer le stockage du flux aléatoire et irrégulier des données permettant de passer des jugements de «terminologisme»?

4) Comment gérer efficacement la motivation du terminologue, surtout lorsque la chute inévitable du rapport nombre de mots dépouillés/nombre de termes relevés commence à se manifester?

5) Comment, enfin, différencier les termes des non-termes ?

La première de ces interrogations renferme deux problèmes, celui de la linéarité des textes et celui même des proportions que pourrait prendre un corpus rassemblé pour le repérage exhaustif des termes d'un domaine. Un corpus, dans ce contexte, est forcément constitué de textes qui se lisent de gauche à droite et du haut de la page vers le bas, en tout cas pour le terminologue humain travaillant sur des langues comme l'anglais et le français. Face à ces problèmes, ce dernier ne peut pas s'empêcher de rechercher des procédures permettant de faire le moins de lecture (linéaire) possible.

S'agissant de la deuxième interrogation, il y a lieu de penser que même si le terminologue ignore les termes qu'il recherche, il a autant de (sous-)buts à atteindre qu'il y a de termes potentiels dans le corpus. Or, s'il ne connaît pas d'entrée les termes et doit se fier entièrement aux «clés» présentes dans les textes pour ses jugements de terminologisme, comme nous le soutiendrons ici, il y a lieu de dire que l'espace problème s'étend sur le corpus entier, quelle qu'en soit la longueur. Il en est ainsi pour deux raisons. Primo, tout terme potentiel recherché par le terminologue naviguant à vue peut tout aussi bien intervenir dans la première ligne du corpus que dans la dernière. Dans la mesure où celui-ci ne peut pas le savoir d'avance, pour un corpus de cent pages, il ne peut théoriquement statuer sur tout terme potentiel $x$ qu'après le parcours des cent pages. Secundo, si le terminologue dépend des clés présentes dans les textes du corpus pour ses jugements de terminologisme et si ces clés peuvent tout aussi bien être rassemblées au même endroit du texte qu'elles peuvent être éparpillées sur l'étendue du corpus, on voit encore que l'espace problème s'étend sur le corpus entier.

Troisième interrogation : comment gérer efficacement le stockage du flux aléatoire et irrégulier des données permettant de passer des jugements de terminologisme ? Le problème posé ici est très étroitement lié au fait, soulevé au paragraphe précédent, que les données nécessaires pour statuer sur une unité sous considération sont très rarement rassemblées au même endroit du texte. Le terminologue doit par conséquent trouver le moyen de gérer non seulement les aléas du flux, mais aussi le stockage des données partielles en attendant qu'elles permettent de trancher dans un sens ou dans un autre.

Interrogation numéro 4 : Comment conserver la motivation initiale du terminologue quand la chute inévitable du rapport nombre de mots dépouillés/nombre de nouveaux termes relevés commence à se manifester? C'est un fait bien connu que plus un texte s'allonge, plus le rapport nombre d'occurrences/nombre de formes se modifie en faveur du premier (Thoiron 1991). Par analogie avec le temps computationnel mesurant le tas que l'ordinateur peut gérer dans la résolution d'un problème sans exploser, on peut parler de «temps motivationnel» pour décrire le temps pendant lequel le termino- 
logue humain peut poursuivre le dépouillement du corpus s'il ne relève pas de nouveaux termes justifiant son effort. Même si ce temps motivationnel est difficile à fixer, l'expérience empirique montre qu'il existe et que le seul problème qui puisse réellement empêcher un repérage exhaustif ne peut être que motivationnel.

\section{LA MÉTAPHORE DU JOUEUR D'ÉCHECS}

Avec ces éclaircissements, quelles sont les améliorations à apporter à notre algorithme de départ pour qu'il garantisse une solution en temps motivationnel ? Il n'y a pas de réponse toute faite mais profitons de l'expérience de la résolution d'un problème semblable au nôtre à bien des égards dans un autre domaine. Il s'agit de la programmation des jeux et, en l'occurrence du jeu d'échecs. L'essentiel de la similitude ici réside dans le fait que c'est un problème dont la résolution passe souvent par la recherche mais on verra plus loin qu'il y a peut-être aussi un intérêt plus psychopédagogique au recours à cette métaphore.

Il y a accord dans la littérature que si un tel programme se contente d'une traversée directe et simple de l'arbre du jeu, il ne parviendra jamais à faire le premier pas et encore moins à atteindre l'état de but dans le temps d'une vie. Car il est estimé que pour un espace de jeu d'un facteur de branchement de 35 et où chaque joueur est appelé à effectuer 50 déplacements, le programme sera appelé à examiner $35^{100}$ de positions différentes (Rich 1983). Le bon sens voudra donc qu'on incorpore dans un tel programme des procédures permettant d'améliorer l'efficacité du moteur de recherche. Au moins deux améliorations se profilent ici. Premièrement, puisque la plupart des procédures de recherche consistent à générer et, ensuite, à évaluer, on pourrait chercher à améliorer le module de génération afin de ne générer que des chemins prometteurs. Deuxièmement, on pourrait aussi chercher à améliorer le module d'évaluation afin de reconnaître et d'explorer en premier les chemins les plus prometteurs. Prises individuellement, ces deux améliorations permettront de réduire l'espace de recherche, mais combinées, elles pourraient le réduire davantage.

\section{QUESTIONS AU PROJET TERMINOLOGIQUE, LE PROJET TERMINOLOGIQUE EN QUESTION}

Nous avons mené la discussion jusqu'ici comme s'il y avait accord sur toutes les questions importantes qui pourraient se poser dans le cadre du présent article et, audelà, du projet terminologique de façon générale : celle de l'utilité du dépouillement de corpus, de la possibilité du métier de terminologue-linguiste, de la distinction entre terminologie générale et terminologie d'application, de la distinction entre terminologie descriptive et terminologie de désignation. Poursuivre la discussion sans tenter de poser ces questions serait, comme le démontrent une bonne partie des critiques qu'on nous a faites depuis que nous travaillons sur les idées exprimées ici, courir le risque de nous acharner sur des questions dont l'utilité n'est pas démontrée.

Ailleurs (Chukwu 1993b), nous avons proposé de considérer qu'il y a pour tout domaine $\mathrm{x}$, la possibilité de deux types de terminologues. Le premier, qu'on appellera terminologue- $x$ ( $x$ étant un tenant lieu pour le domaine envisagé), est terminologue en vertu surtout de son statut de spécialiste du domaine cible. Le second, que nous appelons depuis terminologue-linguiste (désormais terminolinguiste), l'est plutôt en vertu de sa formation dans des principes qui, idéalement, lui permettraient de remplacer n'importe quel terminologue-x sans prétendre être spécialiste du domaine en question. Il nous semble que les questions soulevées par la constitution de la nomenclature du dictionnaire terminologique par le dépouillement de corpus sont plutôt exotiques pour 
les terminologues-x puisque leur statut de spécialistes de domaine fait d'eux des détenteurs des termes de leur domaine au même titre que leurs collègues voués entièrement à des tâches plus orthodoxes. Et, à supposer même qu'un terminologue-x choisisse de passer par un corpus, ce serait moins pour pouvoir en déduire les termes de son domaine que pour en faciliter la remémoration. Ces questions concernent donc le terminolinguiste, puisqu'il ne peut pas constituer la nomenclature qu'il va décrire sans passer par les spécialistes, soit en leur demandant directement les termes employés dans leur spécialité, soit en essayant de déduire ceux-ci par le dépouillment des textes rédigés par ces mêmes spécialistes.

Si on opte pour la seconde solution ${ }^{2}$, comme c'est souvent le cas, on se heurte à deux oppositions possibles. Premièrement, sur quoi se fonde l'espoir du terminolinguiste de pouvoir déduire les termes d'un domaine à partir des textes, sachant que ces termes désignent des configurations de connaissances dont seuls sont détenteurs les spécialistes ? Là, il nous semble qu'on peut répondre en disant que tout terme est à la fois un objet de connaissance (en ce sens qu'il peut recevoir une définition et faire l'objet d'un discours) et un objet d'emploi, donc un élément de communication. C'est, nous semble-t-il, dans ce sens qu'il faut comprendre les propos de Shore (1991), qui soutient que tout symbole connaît deux naissances : la première, privée, lorsque le rapport entre le symbole et le réel est fixé, et la seconde, publique, lorsque le symbole entre dans la communication. S'agissant du terme, on peut considérer que la première naissance correspond au moment de sa définition, étant entendu qu'il ne s'agit pas d'un moment précis, figé à jamais où le rapport entre le terme et le réel serait fixé pour toujours, mais plutôt d'un moment dynamique. La seconde naissance a lieu lors de l'emploi effectif du terme dans des actes de communication. Et là encore, il est clair que nous avons affaire à un acte sans cesse recommencé et qui peut même modifier le rapport du terme avec le réel, donc réenclencher la dynamique de la première naissance. On peut donc imaginer qu'il soit aussi possible de prendre connaissance du statut de terme d'une unité en discours.

Deuxièmement, si le terminolinguiste ne peut pas se passer des spécialistes, pourquoi perdre du temps à dépouiller un corpus et, surtout, pourquoi ne pas reconnaître que la terminologie est l'affaire des spécialistes du domaine ? Nous opterons ici pour une réponse d'ordre économique et, plus précisément, macro-économique. Il ne s'agit pas de ce qui est bon pour telle ou telle corporation mais plutôt du bien de la nation, de la meilleure gestion des ressources de la nation. Une nation développée et, à plus forte raison, une nation en développement ne peut pas se permettre de répartir ses physiciens, par exemple, entre ceux qui poursuivront «les recherches qui changent le monde» et ceux qui se consacreront aux travaux terminologiques du domaine. Bref, il s'agit d'essayer de faire faire à chacun ce qu'il fait le mieux avec le minimum de ressources.

L'autre parti pris qui sous-tend la discussion jusqu'ici est celle de la validité de la distinction faite par Gouadec (1993) entre «terminologie d'application» et «terminologie générale». Cette distinction a suscité une opposition qui, réflexion faite, nous paraît fondée. Elle laisse entendre qu'il pourrait exister une pratique terminologique qui ne soit pas d'application, alors qu'en réalité, l'émergence d'une pratique terminologique autonome a souvent été justifiée en évoquant la nécessité de renoncer à la tentation de tout dire sur tout. Le problème n'est ni simplement terminologique ni simplement sémantique, et il ne suffirait pas de modifier seulement la terminologie ou d'apporter des précisions sémantiques. Faisons plutôt la distinction entre la terminologie appliquée à une spécialité et la terminologie appliquée à une tâche ${ }^{3}$. La première sera entendue au sens de ce qu'on pourrait appeler une terminologie d'école, avec beaucoup de considé- 
rations théoriques, recherchant avant tout la fidélité à un domaine de spécialisation. Elle fera à ce titre partie, avec des disciplines comme l'histoire des sciences, la sociologie des connaissances, l'épistémologie, etc., des disciplines constituant la métascience (voir Frawley 1988; Ziman 1984, ce dernier utilise le terme Science Studies). Si une telle terminologie est théorique, elle pourrait se greffer à l'histoire des sciences (c'est le cas, par exemple, de la monographie de Boisson (à paraître) sur les dénominations de la règle à calcul) ou à la métascience anarchiste au sens de l'étude du partage du pouvoir scientifique (voir par exemple l'étude de Roberts (1991) sur le calorimètre) ou être au service de la didactique des sciences (voir par exemple Thoiron et al., à paraître). Pratique, elle pourrait prendre la forme du Dictionary of Machine Tools de Wüster. La seconde conservera le sens de la terminologie d'application de Gouadec. Selon qu'il s'agit de l'une ou de l'autre, le problème n'a pas la même ampleur. De façon générale, le choix des unités qui feront l'objet d'une description dans ce second cas dépendra très souvent de l'appréciation de la difficulté posée par l'unité compte tenu de la tâche en vue. Ceci est facilement vérifié avec l'application à la traduction. Le traducteur technique qui compile une terminologie retiendra en priorité les unités et expressions (avec ou sans un sens suffisamment spécialisé) qui lui posent des problèmes de traduction.

Nous avons aussi fait usage de la distinction entre terminologie de désignation et terminologie descriptive, distinction jugée importante pour les questions abordées ici. La première serait une pratique terminologique avec une forte part de création de désignations. La seconde, en revanche, serait plutôt une pratique terminologique qui s'apparente à la lexicographie avec ceci de différent qu'elle sera d'application, au sens précisé plus haut. Si on considère que toutes les langues, quelles qu'elles soient, évoluent par des étapes successives qui ne sont pas forcément discrètes, on peut dire que la terminologie pratiquée dans une langue donnée, considérée pour la clarté de la discussion comme la plus évoluée, était d'abord une terminologie de désignation avant de devenir une terminologie descriptive.

Une terminologie de désignation nous paraît symptomatique d'un dysfonctionnement du système (de la langue) et constitue de ce fait un palliatif. Un système qui fonctionne correctement n'a pas besoin qu'on se réunisse dans des commissions pour décider de la manière de désigner tel ou tel objet nouvellement inventé. Donc, dans la mesure où tout système tendrait vers cet état d'équilibre interne qui lui permet d'être autosuffisant, la pratique terminologique dans quelque langue que ce soit tendrait aussi vers une pratique descriptive. Et les questions abordées ici cessent dès lors d'être restreintes à quelques langues très développées comme l'anglais et le français.

\section{AMÉLIORATIONS}

Revenons au problème du repérage des termes à proprement parler en revenant à notre algorithme de départ. Même si les démarches qui y sont reproduites peuvent paraître élémentaires, il n'en est rien en réalité. Nous y avons déjà incorporé des optimisations importantes.

La première de ces optimisations concerne la base où sont stockés les termes sûrs du domaine. Ce n'est pas l'incorporation de celle-ci qui est importante, mais plutôt la décision de ne pas partir avec une base vide. La maigre littérature sur le dépouillement de corpus aux fins de la constitution de nomenclature a souvent insisté à bon escient sur la familiarisation préalable du terminolingusite avec le domaine, même si on n'a jamais été clair sur le but exact de cet exercice et le double emploi qu'il pourrait faire s'il était destiné avant tout à faire acquérir au terminolinguiste des connaissances factuelles de type «x est un terme du domaine». Nous pensons, en revanche, que cet exercice est pri- 
mordial parce qu'il permet de déceler les lois du genre nécessaires aux jugements de terminologisme auxquels le terminolinguiste sera constamment appelé ainsi que les connaissances domainières appelées à encadrer ces jugements. C'est qu'il nous semble que dans le contexte de la problématisation retenue ici, les connaissances procédurales sont supérieures aux connaissances factuelles, et ce pour la simple raison qu'elles peuvent produire ces dernières. Ceci ne revient toutefois pas à dire que le terminolinguiste pourrait partir avec une base de données de termes sûrs vide : c'est une impossibilité. Une telle base est forcément initialisée avec, au moins, un terme sûr dont l'existence est fixée hors corpus parce qu'il constitue le critère de constitution de corpus et le terme premier auquel le terminolinguiste cherchera par la suite à relier les autres unités du corpus pour prouver leur terminologisme. Nous avons proposé d'appeler cette unité «terme a priori» (voir Chukwu 1993b) et elle n'est autre que le nom du domaine.

Une autre amélioration consiste en l'incorporation d'un système de pondération. Bien que l'inclusion d'une unité dans la nomenclature à décrire implique que celle-ci est considérée comme un terme, il serait erroné de penser que le terminolinguiste affirme par là que l'unité appartient à la terminologie du domaine de la même façon qu'on peut affirmer qu'un tel est le fils biologique de telle femme. Tenir une telle position reviendrait à faire complètement abstraction des va-et-vient, des échanges entre disciplines. Ce dispositif de pondération permet alors d'abord d'exprimer le niveau d'intégration d'une unité empruntée à un autre domaine dans le domaine cible. Il est également nécessité par la manière dont le terminolinguiste procède pour constituer la nomenclature de son dictionnaire. Notre sentiment est qu'il se fonde plus sur la présence de clés dont il sera longuement question plus loin. Ces clés étant rarement rassemblées au même endroit du texte, l'affectation d'un coefficient de certitude permettrait donc de tenir compte de l'apport des occurrences successives d'une même unité dans le jugement définitif.

Pour faciliter l'utilisation des coefficients de certitude dans ce second emploi, nous avons aussi, par anticipation, introduit une amélioration notable, à savoir une base de données pour stocker les unités dont le coefficient de certitude ne permet pas encore de se prononcer définitivement.

La troisième amélioration déjà introduite est celle de l'inclusion d'une base de données antidictionnairiques. Nous n'avons pas voulu faire table rase des connaissances du terminolinguiste en tant que sujet-dans-le-monde. Celui-ci, de par son expérience, sait qu'il y a des unités qui, à elles seules, ne peuvent jamais être des termes. De telles unités pourraient être stockées dans cette base. Ainsi, plutôt que de perdre son temps à faire passer de telles unités par la moulinette habituelle, il suffira d'une vérification rapide dans la base pour les écarter.

S'agissant des améliorations à apporter, elles peuvent être réparties entre les deux modules principaux - le module de génération et le module d'évaluation de terminologisme. Au niveau du module de génération, on pourrait opter pour une génération sélective des unités répondant à certains critères: morphologiques, typographiques, fonctionnels, etc. On pourrait aussi, dans certains cas, générer des configurations textuelles entières plutôt que de simples unités et, surtout, le terminolinguiste pourrait chercher à générer des formes plutôt que des occurrences.

Au niveau du module d'évaluation, l'amélioration principale consistera à rendre un peu de souplesse à l'ordre de déclenchement des tests. On pourrait imaginer, par exemple, un système permettant d'assigner des poids à la productivité et à la conclusivité des différents tests selon le domaine. Ainsi, il sera possible de savoir quels tests utiliser en premier. 
Nous proposons d'approfondir les modalités de ces améliorations dans un cadre théorique bien précis que nous avons appelé le pacte terminologique.

\section{LE PACTE TERMINOLOGIQUE}

À défaut d'une définition en bonne et due forme du pacte terminologique, nous avons essayé de relier l'idée à celle de «pacte de référence» de Genette (1972) en passant par celle de «pacte autobiographique» de Lejeune (1975), nous contentant de pratiquer ici et là de l'écriture à la marge pour faire ressortir ce qu'est le pacte terminologique. Tout comme le pacte autobiographique de Lejeune postule que l'autobiographie n'existe que par la triple identité «auteur-narrateur-personnage», c'est-àdire parce qu'un individu avec une existence hors du texte s'engage solennellement à raconter l'histoire de sa vie et rien que l'histoire de sa vie, le pacte terminologique postule que le terme n'existe pour le terminolinguiste parce qu'un spécialiste s'engage, par la signature même de son texte, à employer les termes de sa spécialitét. En empruntant cette formulation, nous avons voulu souligner une distinction fondamentale entre discours technique et discours scientifique. Alors que le premier, même lorsqu'il est signé ce qui n'est pas toujours le cas - est souvent un discours donné tel quel (as is), sans aucune garantie de fiabilité, le discours scientifique met toujours en jeu la réputation, toute l'histoire d'une personne physique ainsi que celle de l'organisme dont il relève (cf. l'histoire de la mémoire de l'eau). En ce sens, alors, on pourrait imaginer une sorte d'entente morale entre le terminolinguiste et le spécialiste du domaine, entente par laquelle le dernier autorise le premier à relever un terme à chaque fois qu'il lui en signale un. On ne peut que regretter l'insuffissance d'une démarche fondée uniquement sur cette première interprétation qui fait du pacte terminologique un pacte posé.

Il faut donc une deuxième interprétation complétant la première mais capable aussi de fonctionner seule, pour des raisons qui deviendront évidentes plus loin. Cette deuxième interprétation repose sur l'appartenance de tout texte de discours de spécialité à un genre - le genre étant défini par la double communauté de contenu et de structure entre des textes (Trimble 1985; Beacco 1992). Si la structure revêt autant d'importance même pour les textes du discours de spécialité dans lesquels la fonction d'information est considérée a priori comme dominante, c'est parce que tout texte, dès lors qu'il relève d'un genre, «fait sens» en partie par son adéquation avec un modèle qui est à la fois masque de rédaction et masque de lecture. C'est dans ce sens que Thoiron (1991) soutient que le texte du discours de spécialité contient la clé de son propre décodage, fait déjà souligné par Latour \& Woolgar (1988 : 79-80) :

Bien que n'ayant pas compris grand-chose au contenu des articles qu'il lisait, il mit au point une technique grammaticale simple permettant de différencier les types d'énoncés scientifiques sans être obligé de s'en remettre aux chercheurs pour élucidation ou assistance.

Nous avons voulu, avec le pacte terminologique, élaborer une grammaire de haut niveau ${ }^{5}$ capable à la fois de générer et de prédire les configurations textuelles susceptibles de contenir des termes ainsi que fournir des tests de terminologisme garantissant la résolution en temps motivationnel du problème du repérage exhaustif des termes. Parce que le discours scientifique est un discours inspirant la confiance, ne serait-ce que par l'engagement d'une personne physique vis-à-vis de sa fiabilité, le pacte terminologique est voulu d'abord comme un pacte posé. Ensuite, compte tenu du fait que chaque texte de spécialité est à la fois un acte individuel et un acte s'inscrivant dans un ensemble (désigné ici genre) dont on peut rechercher les traces de ses lois, le pacte terminologi- 
que est aussi voulu comme une fonction calculée. Par cette seconde interprétation, on introduit la responsabilité du terminolinguiste puisque les clés impliquées dans les calculs ne sont pas forcément des traces d'une activité scripturale en tant que telle mais, le plus souvent, plutôt des traces d'une appropriation interprétative du terminolinguiste.

Mais la seconde interprétation du pacte a une autre fonction capitale relative à la maîtrise du temps motivationnel. Cette fonction est d'ordre psychopédagogique. Les vertus du jeu dans le maintien de la motivation dans des situations d'apprentissage sont bien connues. Parce que chaque cycle de générer-et-tester dans le pacte terminologique constitue une tentative pour «prendre le spécialiste dans son jeu», jeu entendu cette fois-ci littéralement, le travail du terminolinguiste devient un travail-jeu et le choix de la métaphore du joueur d'échecs prend alors tout son sens et on se rapproche de la mâ̂trise complète du temps motivationnel.

À l'échelle du dépouillement manuel, ce que le pacte terminologique propose est un système de balayage rapide (ou lecture diagonale) interrompu seulement par le repérage des indices et de configurations textuelles entières suggérant la présence probable de termes. Une fois de tels indices repérés, le terminoloque peut marquer un arrêt et récupérer l'environnement immédiat pour une étude plus approfondie. De tels indices peuvent prendre la forme de reformulations (définitions contextuelles), de termes métalinguistiques, d'images, de descriptions de concepts, de relations lexicales/sémantiques et conceptuelles, d'effets typographiques de type emploi d'italiques ou de caractères gras, d'abréviations, de ressemblance formelle (morphologie) - la liste ne prétend pas être exhaustive.

\section{APPLICATION}

Compte tenu des contraintes d'espace, nous passerons directement à une application au dépouillement assisté par ordinateur et, à l'occasion, nous démontrerons comment un tel exercice prend pour modèle les démarches du terminologue humain. Pour la même raison que ci-dessus, notre application portera sur un seul facteur, à savoir les abréviations ${ }^{6}$.

\section{Système}

L'application sera réalisée avec TACT, logiciel de génération de concordances. Ce choix est influencé en grande partie par la facile disponibilité du logiciel qui est distribué comme partagiciel (shareware). Mais l'application aurait pu aussi être réalisée avec n'importe quel autre logiciel de génération de concordances (voir, par exemple, van Campenhoudt 1992).

\section{Corpus}

Le corpus est constitué de trois textes tirés de deux revues informatiques françaises - PC Expert et Décision Micro. Ce choix, lui aussi, est motivé. Tout le travail de développement du pacte terminologique a été fait avec des textes du discours scientifique dont la garantie de fiabilité des informations données et du respect des lois régissant le genre repose sur l'engagement d'une personne physique, donc permettant l'exploitation à la fois du pacte posé et du pacte résultant des calculs d'un terminolinguiste-décodeur. En tentant une application sur des textes du discours technique normalement donnés sans une garantie quelconque quant à leur fiabilité, nous avons voulu apporter la preuve que le deuxième aspect du pacte terminologique peut tout aussi bien être complémentaire du premier qu'il peut fonctionner seul. 
Deux des articles (un de chacune des revues) portent sur les écrans des ordinateurs portables et le troisième (tiré de $P C$ Expert) porte sur la carte mémoire PCMCIA. Ce choix a surtout été influencé par le désir de voir s'il existe une continuité (en matière non seulement de terminologie mais aussi des traces constituant les facteurs du pacte terminologique) entre sources différentes et thèmes différents.

Ces textes ont été numérisés et sauvegardés en format ASCII, le seul reconnu par TACT. Cependant, pour compenser les pertes d'informations résultant de cette contrainte, nous avons procédé à une préédition permettant de restituer ce qui pouvait l'être (voir annexe 1 pour un échantillon du corpus avant et après la préédition). Le texte a été ensuite converti en une base de données textuelles TACT avec le module du logiciel prévu à cet effet, et le dépouillement «automatique» réel du corpus a consisté en l'interrogation de la base.

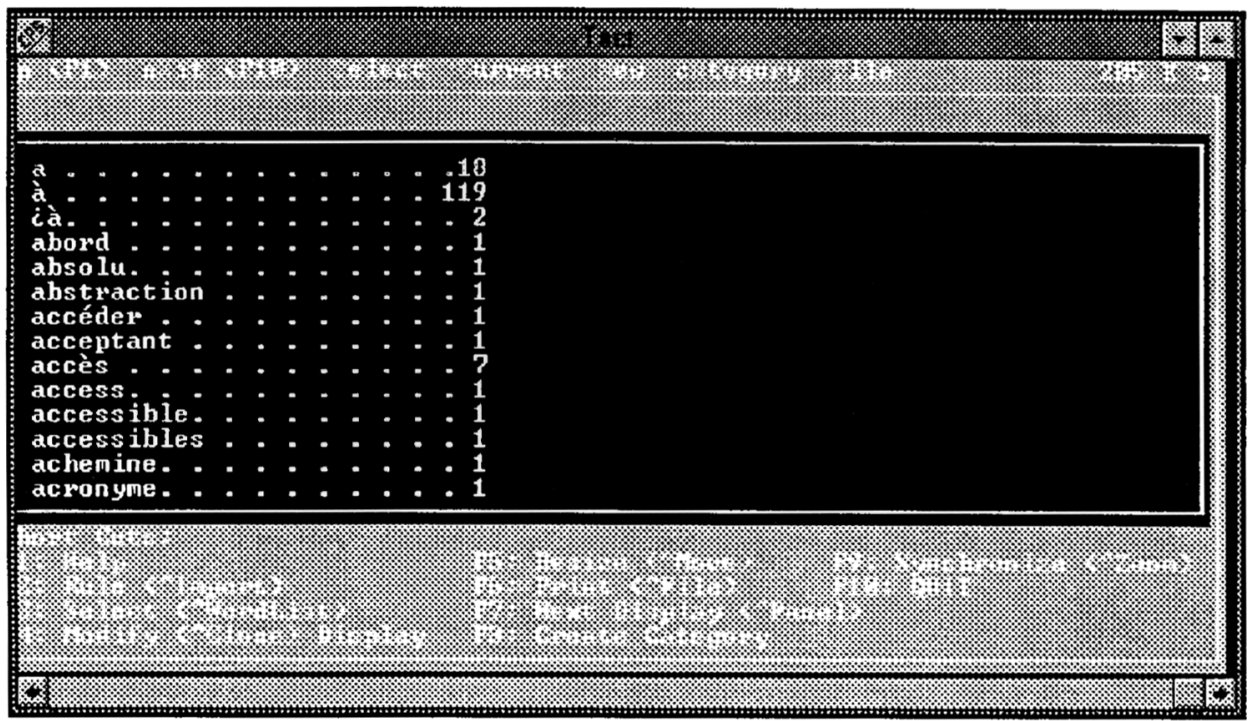

Figure 1

La liste des mots du corpus avec leur fréquence.

Sans avoir encore eu à faire appel au pacte terminologique, on constate (voir figure 1) qu'un certain nombre de problèmes épineux du dépouillement manuel reçoivent un début de réponse avec le recours à un système de repérage automatique ou assisté par ordinateur. Le premier de ces problèmes est celui de la linéarité du langage naturel. Quelles que soient les astuces déployées par le terminolinguiste, sa lecture des textes de son corpus, même quand elle se veut diagonale, reste toujours, ne serait-ce qu'en partie, linéaire. La conversion du texte en une liste permet d'attester beaucoup plus facilement les unités dont il est fait. Il est d'autant plus facile que la liste qui en résulte n'est plus une liste des occurrences, mais plutôt une liste des formes, et l'espace problème s'en trouve considérablement réduit. Les statistiques relatives au texte révèlent qu'au lieu des 4979 occurrences initiales du texte linéaire, nous n'avons que 1509 types organisés dans l'ordre alphabétique. L'absence d'une fonctionnalité de lemmati- 
sation dans le logiciel à ce stade limite sans doute la portée des gains de cette réduction de l'espace problème.

\section{Pacte terminologique et abréviations}

Le fondement psychologique de l'utilisation des abréviations comme facteur de calcul du pacte terminologique dérive des motivations de leur emploi dans le discours spécialisé et le caractère presque iconographique de ces signes qui s'écrivent souvent en majuscules, contrastant ainsi de leur environnement textuel immédiat (voir Chukwu 1993a). Au niveau du dépouillement manuel, ces signes sont faciles à repérer puisqu'ils sont en majuscules. Mais la conversion du texte en une base de données TACT qui ramène les majuscules aux minuscules fait perdre cette iconicité. D'où le traitement que nous leur avons proposé au stade de la préédition, à savoir l'ajout du signe diacritique ' $i$ ' (voir annexe 1) qui leur permet de retrouver leur iconicité ${ }^{7}$. Le terminolinguiste peut ainsi les identifier facilement simplement en consultant la liste des formes de la base.

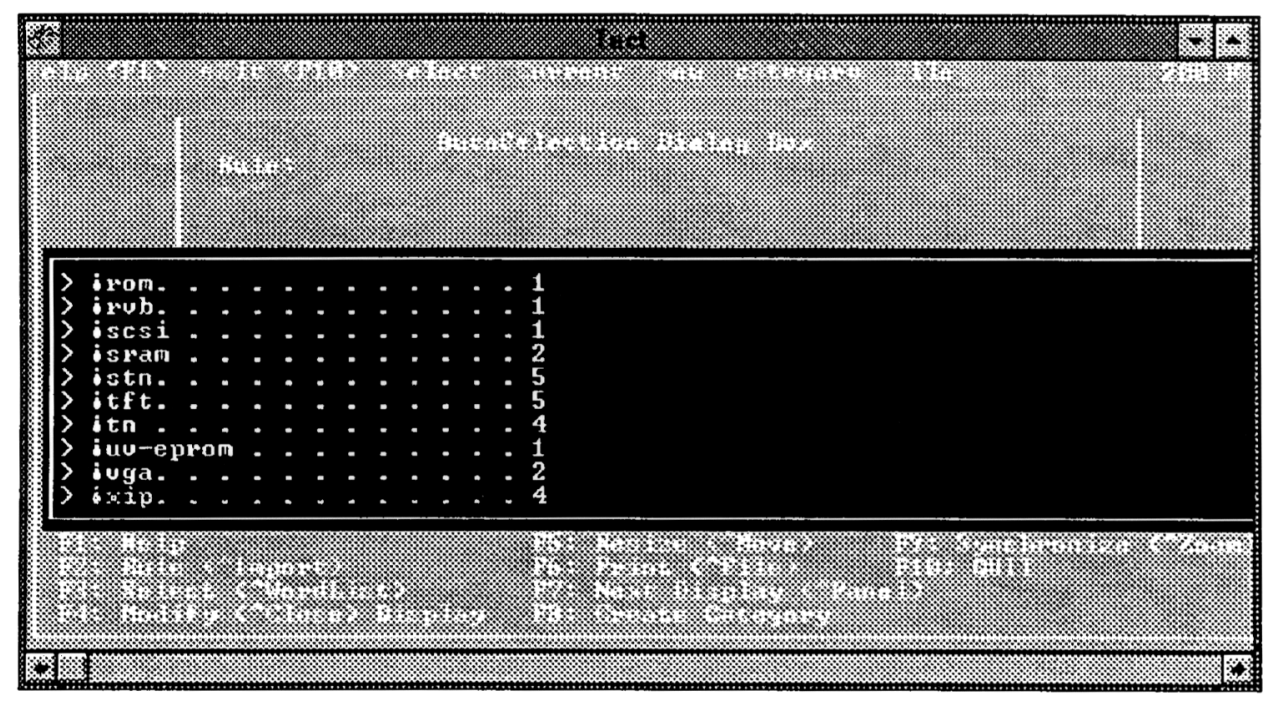

Figure 2

Les sigles du corpus générés à l'aide d'une expression régulière.

Leur aspect particulier (figure 2) fait aussi qu'on peut facilement, à l'aide d'expressions régulières, générer l'ensemble des formes abréviées (c'est-à-dire écrites tout en majuscules) dans le corpus. Le grand avantage d'une telle démarche est que la sous-base générée offre plus de garantie d'une solution en temps motivationnel. Cette garantie est d'autant plus importante ici que le pourcentage de traversées réussies (aboutissant à l'identification d'un nouveau terme) est maximal, l'ensemble des quarante et une unités (en comptant aussi les noms de fabricants tels IBM et NEC) répondant au critère utilisé pour interroger la base s'étant révélés être des termes ${ }^{8}$. 
Puisque la seule présence de sigles, même pour un domaine comme celui de l'informatique où la siglaison est une technique de formation de termes très productive, n'est pas suffisante pour conclure à la présence d'un terme, on pourrait procéder à certains tests de terminologisme pour en avoir le cœur net. Le premier est sans doute la vérification dans un antidictionnaire contenant des abréviations habituelles. Le deuxième consiste à étudier le contexte d'emploi de l'unité pour rechercher d'autres facteurs de création du pacte terminologique, notamment les reformulations. Prenons l'un des sigles présents dans le corpus, en l'occurrence «XIP», pour voir de plus près la manière dont pourrait se dérouler les tests de terminologisme. L'interrogation de la base pour les contextes d'emploi de l'unité (à l'aide de l'affichage KWIC) donne le résultat suivant :

\section{¡xip (4)}

D'autre part, la spécification "¡XIP", acronyme de eXecute In Place, prévoit l'exécution d'un logiciel depuis la carte, dont on adresse directement la mémoire sans avoir à passer par la recopie du logiciel dans la mémoire vive de l'ordinateur hôte.

PCEXP392: 250)

Il en existe trois que l'on trouve $\mathrm{d}^{*}$ 'ores et déjà sur les ordinateurs équipés d'adaptateurs ¡PCMCIA, à savoir ¡FAT (File Allocation Table), ¡XIP (eXecute In Place) et ¡FFS (Flash File System)

(PCEXP392: 254)

Le pilote ¡XIP, lui, se comporte tel un gestionnaire d'¡EMS (Expanded Memory System) pour les architectures 80286 ou antérieures et comme un gestionnaire de mémoire étendue pour les micro-ordinateurs équipés de i386 ou de i486.

(PCEXP392: 254)

L'utilisation de ¡XIP nécessite une adaptation des applications, même mineure dans le cas de logiciels sachant déjà gérer l'¡EMS.

(PCEXP392: 254)

\section{Tableau 1}

Affichage KWIC (mot-clé dans le contexte d'emploi) pour le sigle XIP.

L'examen des deux premiers contextes d'occurrence de l'unité ne laisse aucun doute sur son statut de terme car, comme nous le soutenions dans Chukwu (1993a), avec la prolifération des sigles dans la terminologie et le discours de la plupart des spécialités, de plus en plus de définitions seront représentées, entièrement ou seulement en partie, «X sigle (acronyme, abréviation, etc.) de Y». L'expansion d'une forme abrégée doit, par conséquent, être considérée comme une reformulation. Il faut également souligner que le statut reformulatoire de l'expansion d'une abréviation est par rapport aux deux plans ontologique (elle établit l'existence d'un terme puisqu'une abréviation qui n'est pas terme ne pourra pas appeler à une expansion) et épistémologique (elle donne des renseignements sur le terme et en réduit l'opacité $)^{9}$. Nous ajouterons que l'introduction du syntagme sous-jacent au sigle ici est amplement justifiée parce que l'une des voies favorites de décodage du sigle est par la reconstitution du syntagme sous-jacent. Il se trouve ici qu'à moins d'être au fait des tendances actuelles en la matière, la reconstitution du syntagme dans cet exemple doit poser des problèmes 
quasi insolubles : le choix de la lettre ' $\mathrm{X}^{\prime}{ }^{10}$ plutôt que le 'E' qui commence réellement «eXecute» et l'inclusion de l'initiale d'un mot outil («In»).

Un autre avantage qui découle de la démarche décrite ici, qui permet de rassembler l'ensemble des occurrences (et les contextes) de l'unité dont le terminologisme est en question, est que le problème de la gestion du flux aléatoire et irrégulier des données permettant de passer des jugements de terminologisme cesse d'en être un. Ainsi, nous sommes dispensés de tenir une base de données temporaire pour le stockage des unités dont le coefficient de certitude ne permet pas encore de statuer définitivement sur leur statut.

\section{CONCLUSION}

Au-delà du but avoué de la discussion menée ici de montrer l'une des possibilités d'aborder un problème fondamental dont tout le monde reconnaît la primordialité mais qu'on passe malheureusement souvent sous silence, nous pensons aussi qu'elle pose le problème de la définition du «terme» et de la terminologie comme pratique. Car si une tentative pour résoudre le problème du repérage des termes dans un corpus part forcément de la définition de l'unité «terme», elle se doit aussi d'aboutir à une tentative pour réinventer la définition de celui-ci, ne serait-ce que pour le terminolinguiste qui, a priori, ignore souvent la configuration de connaissances recouverte par le concept désigné par un terme sous considération. L'expérience semble indiquer qu'une définition opératoire du «terme» pour le terminolinguiste se doit de prendre beaucoup plus d'appui sur une conception du terme-dans-le-texte-de-spécialité. Il y a là sans doute une raison qui plaide en faveur d'un plus grand intérêt de la part des terminolinguistes dans le fonctionnement des textes de spécialité.

UZOMA CHUKWU

Montery Institute of International Sutdies, Montery, États-Unis

\section{Notes}

1. Ce n'est pas de la provocation que de dire qu'un problème qui ne peut pas être résolu dans le temps imparti est simplement un problème qui ne peut pas être résolu. Une autre façon de dire la même chose serait de dire que n'importe qui résoudrait n'importe quel problème dans un univers dont le temps n'est pas une composante.

2. Nous excluons d'emblée la première option à cause de la fiabilité douteuse des données d'introspection des sujets sur leur état mental et, par là, sur leurs connaissances.

3. Pour nous, cette distinction ne recouvre pas celle qu'on essaie depuis des années d'instaurer entre «terminologie» et «terminographie» (voir Rey 1975). Car, qu'il s'agisse de la terminologie appliquée à une spécialité ou de la terminologie appliquée à une tâche, elle peut toujours comporter une volée théorique et une volée pratique au sens de l'élaboration de glossaires et de dictionnaires spécialisés.

4. On voit là notre dette à Guilbert (1973: 13), qui écrit à propos du mode de signification du terme : «Le terme scientifico-technique ne peut être dissocié de sa fonction sociale, de la personnalité du locuteur spécialiste». (Voir aussi le texte plus récent de Hermans (1991).)

5. L'opposition, ici, est par rapport à «une grammaire conceptuelle» à laquelle pourrait faire appel un terminologue- $x$ confronté au problème de repérage des termes dans un corpus.

6. Pour une application au dépouillement manuel et portant sur l'ensemble de nos facteurs de calcul du pacte terminologique, voir Chukwu (1993b).

7. Il nous a semblé que si l'on veut être en droit d'exiger de la machine les mêmes performances que l'homme dans des tâches comme celle dont il est ici question, il faut faire en sorte que la machine dispose des mêmes données de base.

8. Il faut toutefois faire remarquer que la productivité très élevée ici n'est pas particulièrement étonnante pour qui fréquente le discours du domaine qui fait un usage important de la siglaison comme technique de formation des termes. Il ne saurait donc être question de s'attendre à une productivité aussi élevée dans d'autres domaines. 
9. Bien évidemment, dans le cas précis discuté ici, on pourrait se demander si l'expansion œuvre réellement dans le sens de la réduction de l'opacité, puisqu'elle aboutit à un syntagme en anglais dans un texte français. La fonction qui prédomine ici est donc celle de l'établissement du statut de terme de l'unité considérée.

10. Ce choix est à comparer avec celui qui est opéré dans ExCA (pour Exchangeable Card Architecture) dans le corpus.

\section{RÉFÉRENCES}

BEACCO, Jean-Claude (1992): «Les genres textuels dans l'analyse du discours: écriture légitime et communautés translangagières», Langages, 105, pp. 8-27.

BEAUGRANDE, R. de and W. DRESSLER (1981): Introduction to Text Linguistics, London, Longman.

BEJOINT, Henri (1993) : «La définition en terminologie», P. J. L. Arnaud et Ph. Thoiron (dir.), Aspects du vocabulaire, Lyon, Presses Universitaires de Lyon, pp. 18-25.

BOISSON, Claude Pierre (à paraître) : «Les dénominations de la règle à calcul», monographie, Séminaires du CRTT, Université Lumière Lyon-2.

CHUKWU, Uzoma (1993a) : «La terminologie de l'informatique : à la recherche de clés d'accès», P. J. L. Arnaud et Ph. Thoiron (dir.), Aspects du vocabulaire, Lyon, Presses Universitaires de Lyon, pp. 27-48.

CHUKWU, Uzoma (1993b) : Le repérage des termes dans un corpus bilingue anglais/français, Thèse de doctorat nouveau régime, Université Lumière Lyon-2.

FRAWLEY, William (1988): «Relational Models and Metascience», Martha W. Evens (Ed.), Relational Models of the Lexicon. Representing Knowledge in Semantic Networks, Cambridge, Cambridge University Press, pp. 335-372.

GENETTE, Gérard (1972) : Figures III, Paris, Éditions du Seuil, coll. «Poétique».

GOUADEC, Daniel (1993) : Terminologie et terminotique. Outils, modèles et méthodes, Actes de la première Université d'automne en terminologie, Université de Rennes-2, 21-26 septembre 1992, Paris, La Maison du dictionnaire.

GUILBERT, Louis (1973) : «La spécificité du terme scientifique et technique», Langue.

HERMANS, A. (1989) : «La Définition des termes scientifiques», Meta, 34 (3), Montréal, pp. 529-538.

LATOUR, Bruno et Steve WOOLGAR (1988) : La vie de laboratoire. La production des faits scientifiques, Paris, Éditions La Découverte.

LEJEUNE, Philippe (1975) : Le pacte autobiographique, Paris, Seuil, coll. «Poétique».

REY, Alain (1975) : «Terminologies et terminographies», La Banque des mots, 10, pp. 145-154.

RICH, Eliane (1983) : Artificial Intelligence, Tokyo, McGraw-Hill.

ROBERTS, Lissa (1991) : «A Word and the World. The Significance of Naming the Calorimeter», Isis, 82, pp. 198-222.

SAGER, Juan C. (1990) : A Practical Course in Terminology Processing, Amsterdam, John Benjamins.

SHORE, Bradd (1991) : «Twice-Born, Once Conceived: Meaning Construction and Cultural Cognition», American Anthropologist, 93 (1), pp. 9-27.

THOIRON, Philippe (1991): «Texte spécialisé et non-spécialiste : un problème de stratégie de décodage», Revue belge de philologie et d'histoire, 69 (3), pp. 629-643.

THOIRON, Philippe et al. (à paraître) : «Terminologies multilingues et notion d'"archi-concept" : quelques aspects de la recherche en terminologie», Séminaires du CRTT, Université Lumière Lyon-2.

TRIMBLE, Louis (1985) : English for Science and Technology. A Discourse Approach, Cambridge, Cambridge University Press.

VAN CAMPENHOUDT, Marc (1992): «Une norme de dépouillement terminologique en langue française», Équivalences, 21 (1-2), pp. 121-136.

ZIMAN, John (1984) : An Introduction to Science Studies, Cambridge, Cambridge University Press.

\section{ANNEXE 1}

\section{EXTRAIT DE CORPUS AVANT ET APRÈS PRÉÉDITION}

\section{Décision Micro, $\mathrm{n}^{\circ}$ 80, 1992, p. 39}

\section{La Suprématie des écrans LCD}

La plupart des écrans couleurs pour portables repose, aujourd'hui, sur la technologie des cristaux liquides[...]

$[\ldots]$ 
Les cristaux utilisés, appelés «nématiques en hélice» («Twisted Nematic», ou TN), ont pour propriété d'assurer une rotation de l'axe du premier polariseur de 90 degrés avant que la lumière n'atteigne le second. [...]

[...]

De nombreux risques de défauts à la fabrication

$[\ldots]$

PC Expert, $\mathrm{n}^{\circ} 3,1992$, p. 103

Les Notebooks : Technologie des écrans

Les deux principales technologies utilisées sur les notebooks et sur les ordinateurs portables sont les écrans à cristaux liquides (Liquid Crystal Display ou LCD) et les écrans à plasma. $[\ldots]$

$[\ldots]$

PC Expert, $\mathrm{n}^{\circ} 3,1992$, p. 249

\section{Le standard PCMCIA}

$[\ldots]$

$<$ SOURCE DEC8092><pg 39>

$<$ TART La Suprématie des Ecrans LCD >

LA SUPREMATIE DES ECRANS ¡LCD

$<$ Chapeau open $>$ La plupart des écrans couleurs pour portables repose, aujourd*'hui, sur la technologie des cristaux liquides $[\ldots]<$ chapeau close $>$

$[\ldots]$

//

Les cristaux utilisés, appelés «nématiques en hélice» <pth open>(«Twisted Nematic», ou $\lceil\mathrm{TN})<$ pth close $>$, ont pour propriété d'assurer une rotation de l'axe du premier polariseur de 90 degrés avant que la lumière n'atteigne le second. [...]

$[\ldots]$

$<$ STITRE De nombreux risques de défauts à la fabrication>

De nombreux risques de défauts à la fabrication

$[\ldots]$

$<$ SOURCE PCEXP392 ><pg 103>

$<$ TART LES NOTEBOOKS: TECHNOLOGIE DES ECRANS >

LES NOTEBOOKS : TECHNOLOGIE DES ECRANS

Les deux principales technologies utilisées sur les ¿notebooks et sur les ordinateurs portables sont les écrans à cristaux liquides <pth open $>(¿$ Liquid ¿Crystal ¿Display ou ¡LCD) $<$ pth close $>$ et les écrans à plasma. [...]

[...]

$<$ SOURCE PCEXP392 $><$ pg 249 $><$ TART Le standard ¡PCMCIA $>$

$[\ldots]$

\section{ANNEXE 2}

\section{EXTRAIT DES CONCORDANCES POUR LES SIGLES DU CORPUS}

¡ansi (1)

(PCEXP392: 249) | traditionnels l'>iANSI, l'iEIA, l'iISO ou ¡ast (1)

(DEC8092: 39) qu'ont choisi Dell et $>$ ¡AST pour équiper leurs ¡bios (2)

(PCEXP392: 250) | constructeurs. Les $>$ ¡BIOS reconnaissant les

(PCEXP392: 254) directement dans le $>$ iBIOS des $i P C$, comme une iccit (1) 
(PCEXP392: 249) l';EIA, l'iIS0 ou le $>_{i}$ CCIT, passent | parfois ¡cd1 (1)

(PCEXP392: 250) à cet effet | (">iCD1" et "iCD2", en position ¡cd2 (1)

(PCEXP392: 250) cet effet | (" $i C D 1 "$ et ">iCD2", en position 36 et icis (6)

(PCEXP392: 250) la carte est de lire le $>_{i}$ CIS | (Card Information (PCEXP392: 250) on y stocke, | outre le $>_{i}$ CIS des registres de

(PCEXP392: 252)contenus dans le $>i$ CIS doivent commencer (PCEXP392: 252)est stockée dans le $>_{i}$ CIS. Comme I dans les (PCEXP392: 254) | // | Le lecteur de $>_{i}$ CIS, comme son nom (PCEXP392: 254) contenues dans le $>_{i}$ CIS. Le gestionnaire de ¡dma (1)

(PCEXP392: 250) 64 Mo, accessibles en | >iDMA (pour Direct Memory idos (2)

(PCEXP392: 249) des | compatibles sous >iDOS, son architecture tant

(PCEXP392: 254$) \mid$ son système sous $>_{i}$ DOS ou $¡$ OS $/ 2$ en changeant ¡dram (1)

(PCEXP392: 250)toutefois de la | >¡DRAM, laquelle nécessite ¡dstn (4)

(DEC8092: 39)Twisted | Nematic) et >iDSTN (Double Super

(PCEXP392: 103) | ¿Twisted ¿Nematic (>iDSTN) employé pour | cette

(PCEXP392: 103) technologie. Les écrans $>i$ DSTN ont un niveau de

(PCEXP392: 103) | avec les écrans ¡STN ou >iDSTN. | // | | | LES ¡eeprom (2)

(PCEXP392: 250) une moindre mesure, les $>$ ¡EEPROM sont utilisées en

(PCEXP392: 254) pour | effacer des >iEEPROM ou des Flash ¡EPROM. | eia (1)

(PCEXP392: 249) traditionnels l';ANSI, l'>iEIA, l'iISO ou le ¡CCIT, ¡ems (2)

(PCEXP392: 254) tel un gestionnaire d'>iEMS (Expanded | Memory

(PCEXP392: 254)sachant déjà gérer l'>iEMS. Enfin, ¡FFS, | comme eprom (3)

(PCEXP392: 250) de caractères; les Flash > $>$ EPROM | et, dans une moindre (PCEXP392: 250) disque dur en Flash | $>_{i}$ EPROM, pour ne citer que

(PCEXP392: 254) des ¡EEPROM ou des Flash >¡EPROM. | // | Le lecteur de ¡exca (4)

(PCEXP392: 250) de ¡PCMCIA, appelée >iExCA (pour | Exchangeable

(PCEXP392: 250)Card Architecture). $>i$ ExCA est donc une |

(PCEXP392: 250) les spécifications $\mid$ de $>i E x C A$ et de les mettre à

(PCEXP392: 250) sont | compatibles avec $>_{i}$ ExCA. De sorte que cette ifat (2)

(PCEXP392: 254) iPCMCIA, à savoir >iFAT | (File Allocation

(PCEXP392: 254)Enfin, ¡FFS, | comme >iFAT, est un système de iffs (3)

(PCEXP392: 254)In | Place) et >iFFS (Flash File

(PCEXP392: 254) déjà gérer l'iEMS. Enfin, >iFFS, | comme ¡FAT, est un

(PCEXP392: 254)à son aîné, | >iFFS est spécifiquement 